\title{
Time-Domain Analytical Expression for Near Fields of Arbitrarily Oriented Electric Dipole and Its Application
}

\author{
Qian Yang, ${ }^{1,2}$ Bing Wei, ${ }^{1,2}$ Xinbo He, ${ }^{1,2}$ and Minghao Gong ${ }^{1,2}$ \\ ${ }^{1}$ School of Physics and Optoelectronic Engineering, Xidian University, Xian 710071, China \\ ${ }^{2}$ Collaborative Innovation Center of Information Sensing and Understanding, Xidian University, \\ Xian 710071, China
}

Correspondence should be addressed to Qian Yang; zijiangy@126.com

Received 23 November 2016; Revised 26 April 2017; Accepted 23 May 2017; Published 16 July 2017

Academic Editor: Xianming Qing

Copyright (C) 2017 Qian Yang et al. This is an open access article distributed under the Creative Commons Attribution License, which permits unrestricted use, distribution, and reproduction in any medium, provided the original work is properly cited.

\begin{abstract}
The near fields of electric dipole are commonly used in wide-band analysis of complex electromagnetic problems. In this paper, we propose new near field time-domain expressions for electric dipole. The analytical expressions for the frequency-domain of arbitrarily oriented electric dipole are given at first; next we give the time-domain expressions by time-frequency transformation. The proposed expressions are used in hybrid TDIE/DGTD method for analysis of circular antenna with radome. The accuracy of the proposed algorithm is verified by numerical examples.
\end{abstract}

\section{Introduction}

The electric dipole is an important unit in electromagnetism; its analytical expressions of the radiation field have been described in a number of works, for example, the expressions of special oriented electric dipole [1-3] and the expressions of arbitrarily oriented electric dipole by using the far-field approximation [4]. But the time-domain expressions of arbitrarily oriented electric dipole are necessary to analyze the time-domain near fields problems. The near field is governed by several type fields, the relationship between $\mathbf{E}$ and $\mathbf{H}$ becomes very complex, and special care must be taken when dealing with near fields problems.

The hybrid method takes advantage of several methods and is often used to analyze multiscale electromagnetic problems [5-7], like the shortwave antenna in complex environment [8]. In this study, the analytical time-domain expressions of arbitrarily oriented electric dipole are proposed and used in hybrid TDIE/DGTD method for analysis of circular antenna with radome. The numerical results verify the validity of our algorithm.

\section{The Frequency-Domain Near Fields for an Arbitrarily Oriented Electric Dipole}

Suppose an electric dipole which along $\widehat{\mathbf{r}}_{0}$ is located at original point; the polar angle and the azimuthal angle of $\widehat{\mathbf{r}}_{0}$ are $\theta_{0}$ and $\varphi_{0}$, as Figure 1(a) shows. The observation point is located at $P$, and the coordinates of point $P$ are $r, \theta$, and $\varphi$, respectively, as Figure 1(b) shows.

The magnetic vector potential produced by the electric current in an infinite medium can be written as

$$
\mathbf{A}(r)=\mu \iiint_{v} \mathbf{J}\left(\mathbf{r}^{\prime}\right) \frac{\exp \left(i k\left|\mathbf{r}-\mathbf{r}^{\prime}\right|\right)}{4 \pi R} d v^{\prime},
$$

where $\mu, \mathbf{r}^{\prime}, \mathbf{r}, R$, and $k$ are the permeability, the position vector of the integrating point, the position vector of the observation point $\mathbf{P}$, the distance from the integrating point to the observation point, and the wave number, respectively, as Figure 1(c) shows. 


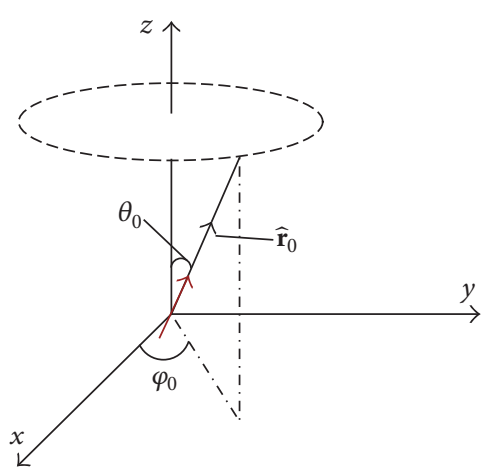

(a) An electric dipole at the original point

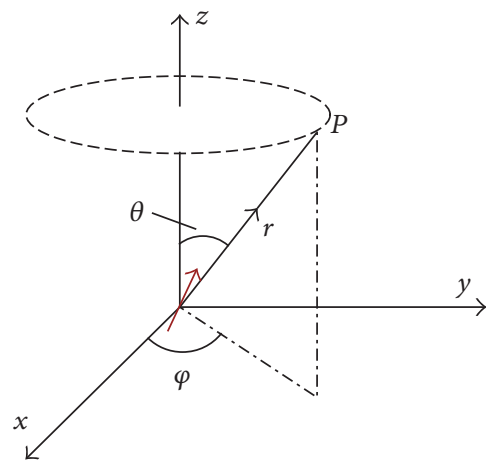

(b) The observation point $(r, \theta, \varphi)$

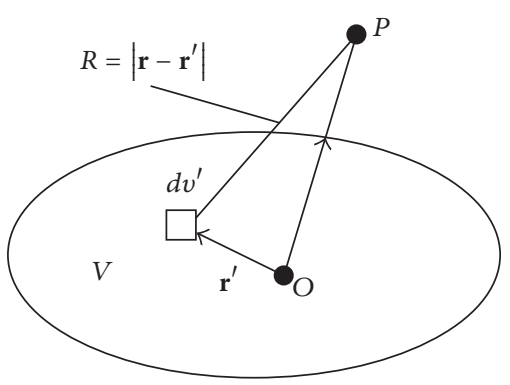

(c) Integrating point and observation point

FIGURE 1: The arbitrarily oriented electric dipole.

A short current source $\widehat{\mathbf{r}}_{0} I l$ can be seen as an electric dipole; considering the characteristic of the $\delta\left(\mathbf{r}^{\prime}\right)$ function, we have

$$
\begin{aligned}
\mathbf{A}(r) & =\mu \iiint_{v} \widehat{\mathbf{r}}_{0} I l \delta\left(\mathbf{r}^{\prime}\right) \frac{\exp \left(i k\left|\mathbf{r}-\mathbf{r}^{\prime}\right|\right)}{4 \pi R} d v^{\prime} \\
& =\widehat{\mathbf{r}}_{0} I l \mu \frac{\exp (i k r)}{4 \pi r},
\end{aligned}
$$

where $\widehat{\mathbf{r}}_{0}$ is defined as

$$
\widehat{\mathbf{r}}_{0}=\widehat{\mathbf{x}} \sin \theta_{0} \cos \varphi_{0}+\widehat{\mathbf{y}} \sin \theta_{0} \sin \varphi_{0}+\widehat{\mathbf{z}} \cos \theta_{0},
$$

where the $\widehat{\mathbf{x}}, \widehat{\mathbf{y}}$, and $\widehat{\mathbf{z}}$ are the unit vector of the rectangular coordinate system. Magnetic field can be evaluated as

$$
\begin{aligned}
\mathbf{H} & =\frac{1}{\mu} \nabla \times \mathbf{A}=\frac{1}{\mu} \nabla \times\left[\widehat{\mathbf{r}}_{0} I l \mu \frac{\exp (i k r)}{4 \pi r}\right]=\frac{1}{\mu} \nabla \\
& \times\left[\left(\widehat{\mathbf{x}} \sin \theta_{0} \cos \varphi_{0}+\widehat{\mathbf{y}} \sin \theta_{0} \sin \varphi_{0}+\widehat{\mathbf{z}} \cos \theta_{0}\right) I l \mu\right. \\
& \left.. \frac{\exp (i k r)}{4 \pi r}\right]=\frac{I l}{4 \pi}\left[\sin \theta_{0} \cos \varphi_{0} \nabla \times\left(\widehat{\mathbf{x}} \frac{\exp (i k r)}{r}\right)\right. \\
& +\sin \theta_{0} \sin \varphi_{0} \nabla \times\left(\widehat{\mathbf{y}} \frac{\exp (i k r)}{r}\right)+\cos \theta_{0} \nabla \\
& \left.\times\left(\widehat{\mathbf{z}} \frac{\exp (i k r)}{r}\right)\right], \\
\nabla & \times \mathbf{A}=\widehat{\mathbf{x}}\left(\frac{\partial A_{z}}{\partial y}-\frac{\partial A_{y}}{\partial z}\right)+\widehat{\mathbf{y}}\left(\frac{\partial A_{x}}{\partial z}-\frac{\partial A_{z}}{\partial x}\right) \\
& +\widehat{\mathbf{z}}\left(\frac{\partial A_{y}}{\partial x}-\frac{\partial A_{x}}{\partial y}\right)
\end{aligned}
$$

which implies curl elements of (4) can be rewritten as

$$
\begin{aligned}
\nabla & \times\left(\widehat{\mathbf{x}} \frac{\exp (i k r)}{r}\right) \\
& =(\widehat{\mathbf{y}} \cos \theta-\widehat{\mathbf{z}} \sin \theta \sin \varphi)\left(\frac{i k}{r}-\frac{1}{r^{2}}\right) \exp (i k r), \\
\nabla & \times\left(\widehat{\mathbf{y}} \frac{\exp (i k r)}{r}\right) \\
& =(\widehat{\mathbf{z}} \sin \theta \cos \varphi-\widehat{\mathbf{x}} \cos \theta)\left(\frac{i k}{r}-\frac{1}{r^{2}}\right) \exp (i k r), \\
\nabla & \times\left(\widehat{\mathbf{z}} \frac{\exp (i k r)}{r}\right) \\
& =(\widehat{\mathbf{x}} \sin \theta \sin \varphi-\widehat{\mathbf{y}} \sin \theta \cos \varphi)\left(\frac{i k}{r}-\frac{1}{r^{2}}\right) \exp (i k r) .
\end{aligned}
$$

Substituting (6) into (2), we get expression of magnetic field for the frequency-domain of an arbitrarily oriented electric dipole

$$
\begin{aligned}
\mathbf{H} & =\frac{i k I l \exp (i k r)}{4 \pi r}\left[\widehat { \mathbf { x } } \left(\cos \theta_{0} \sin \theta \sin \varphi\right.\right. \\
& \left.-\sin \theta_{0} \sin \varphi_{0} \cos \theta\right)+\widehat{\mathbf{y}}\left(\sin \theta_{0} \cos \varphi_{0} \cos \theta\right. \\
& \left.-\cos \theta_{0} \sin \theta \cos \varphi\right)+\widehat{\mathbf{z}}\left(\sin \theta_{0} \sin \varphi_{0} \sin \theta \cos \varphi\right. \\
& \left.\left.-\sin \theta_{0} \cos \varphi_{0} \sin \theta \sin \varphi\right)\right]\left(1+\frac{i}{k r}\right) .
\end{aligned}
$$

The expression of electric field is

$$
\begin{aligned}
\mathbf{E} & =\frac{-i}{\omega \varepsilon} \nabla \times \mathbf{H}=\frac{-i}{\omega \mu \varepsilon} \nabla \times(\nabla \times \mathbf{A}) \\
& =\frac{-i}{\omega \mu \varepsilon}\left[\nabla^{2} \mathbf{A}-\nabla(\nabla \cdot \mathbf{A})\right]=\frac{i}{\omega \mu \varepsilon}\left[k^{2} \mathbf{A}+\nabla(\nabla \cdot \mathbf{A})\right]
\end{aligned}
$$


substituting (2) into (8), we have

E

$$
\begin{aligned}
& =\frac{i}{\omega \mu \varepsilon}\left[k^{2} \widehat{\mathbf{r}}_{0} I l \mu \frac{\exp (i k r)}{4 \pi r}+\nabla\left(\nabla \cdot \widehat{\mathbf{r}}_{0} I l \mu \frac{\exp (i k r)}{4 \pi r}\right)\right] \\
& =\frac{i I l \mu}{4 \pi \omega \mu \varepsilon}\left[k^{2} \widehat{\mathbf{r}}_{0} \frac{\exp (i k r)}{r}+\nabla\left(\nabla \cdot \widehat{\mathbf{r}}_{0} \frac{\exp (i k r)}{r}\right)\right],
\end{aligned}
$$

where

$$
\begin{aligned}
\nabla\left(\nabla \cdot \widehat{\mathbf{r}}_{0} \frac{\exp (i k r)}{r}\right) \\
=\sin \theta_{0} \cos \varphi_{0} \nabla\left(\nabla \cdot \widehat{\mathbf{x}} \frac{\exp (i k r)}{r}\right) \\
+\sin \theta_{0} \sin \varphi_{0} \nabla\left(\nabla \cdot \widehat{\mathbf{y}} \frac{\exp (i k r)}{r}\right) \\
+\cos \theta_{0} \nabla\left(\nabla \cdot \widehat{\mathbf{z}} \frac{\exp (i k r)}{r}\right) .
\end{aligned}
$$

\section{The Time-Domain Near Fields for an Arbitrarily Oriented Electric Dipole}

An electric dipole consists of a positive charge $+q$ and an negative charge $-q$, distance $l$ apart. The dipole moment $\mathbf{p}=$ $q \mathbf{l}$, so that electric current can be represented as

$$
I \mathbf{l}=-i \omega \mathbf{p} \text {. }
$$

Substituting (11) into (7),

$$
\begin{aligned}
\mathbf{H}(\mathbf{r}, \omega)=\frac{\omega^{2} p \exp (i k r)}{4 \pi c r}\left(1+\frac{i c}{\omega r}\right) \\
\cdot\left[\widehat{\mathbf{x}}\left(\cos \theta_{0} \sin \theta \sin \varphi-\sin \theta_{0} \sin \varphi_{0} \cos \theta\right)\right. \\
+\widehat{\mathbf{y}}\left(\sin \theta_{0} \cos \varphi_{0} \cos \theta-\cos \theta_{0} \sin \theta \cos \varphi\right) \\
+\widehat{\mathbf{z}}\left(\sin \theta_{0} \sin \varphi_{0} \sin \theta \cos \varphi\right. \\
\left.\left.\quad-\sin \theta_{0} \cos \varphi_{0} \sin \theta \sin \varphi\right)\right] .
\end{aligned}
$$

The time-frequency conversion relation and the flourier transformation are

$$
\begin{aligned}
-i \omega & \longrightarrow \frac{\partial}{\partial t}, \\
p(t) & =\frac{1}{2 \pi} \int p(\omega) \exp (-i \omega t) d \omega, \\
p\left(t-\frac{r}{c}\right) & =\frac{1}{2 \pi} \int p(\omega) \exp (i k r) \exp (-i \omega t) d \omega .
\end{aligned}
$$
(13)

The time-domain expression of (12) can be obtained by

$$
\begin{aligned}
\mathbf{H}(\mathbf{r}, t)=-\frac{1}{4 \pi c r}\left(\frac{\partial^{2}}{\partial t^{2}}+\frac{c}{r} \frac{\partial}{\partial t}\right) p\left(t-\frac{r}{c}\right) \\
\cdot\left[\widehat{\mathbf{x}}\left(\cos \theta_{0} \sin \theta \sin \varphi-\sin \theta_{0} \sin \varphi_{0} \cos \theta\right)\right. \\
+\widehat{\mathbf{y}}\left(\sin \theta_{0} \cos \varphi_{0} \cos \theta-\cos \theta_{0} \sin \theta \cos \varphi\right) \\
+\widehat{\mathbf{z}}\left(\sin \theta_{0} \sin \varphi_{0} \sin \theta \cos \varphi\right. \\
\left.\left.-\sin \theta_{0} \cos \varphi_{0} \sin \theta \sin \varphi\right)\right] .
\end{aligned}
$$

The time-domain expression of electric field can be obtained in the same way

$$
\begin{aligned}
& E_{x}=\left\{\operatorname { s i n } \theta _ { 0 } \operatorname { c o s } \varphi _ { 0 } \left[A_{1} \cdot \sin ^{2} \theta \cos ^{2} \varphi-A_{2}\right.\right. \\
& \left.\cdot\left(\cos ^{2} \theta \cos ^{2} \varphi+\sin \theta \sin ^{2} \varphi\right)-\frac{\partial^{2}}{\partial t^{2}}\right]+\sin \theta_{0} \sin \varphi_{0} \\
& \cdot\left[A_{1} \cdot \sin ^{2} \theta \sin \varphi \cos \varphi-A_{2}\right. \\
& \left.\cdot \sin \varphi \cos \varphi\left(\cos ^{2} \theta-\sin \theta\right)\right]+\cos \theta_{0}\left[A_{1}\right. \\
& \left.\left.\cdot \sin \theta \cos \theta \cos \varphi+A_{2} \cdot \sin \theta \cos \theta \cos \varphi\right]\right\} \frac{\mu}{4 \pi r} \\
& p\left(t-\frac{r}{c}\right) \\
& E_{y}=\left\{\operatorname { s i n } \theta _ { 0 } \operatorname { c o s } \varphi _ { 0 } \left[A_{1} \cdot \sin ^{2} \theta \cos \varphi \sin \varphi-A_{2}\right.\right. \\
& \left.\cdot \sin \varphi \cos \varphi\left(\cos ^{2} \theta-\sin \theta\right)\right]+\sin \theta_{0} \sin \varphi_{0}\left[A_{1}\right. \\
& \cdot \sin ^{2} \theta \sin ^{2} \varphi-A_{2} \cdot\left(\cos ^{2} \theta \sin ^{2} \varphi+\sin \theta \cos ^{2} \varphi\right) \\
& \left.-\frac{\partial^{2}}{\partial t^{2}}\right]+\cos \theta_{0}\left[A_{1} \cdot \sin \theta \cos \theta \sin \varphi+A_{2}\right. \\
& \cdot \sin \theta \cos \theta \sin \varphi]\} \frac{\mu}{4 \pi r} p\left(t-\frac{r}{c}\right), \\
& E_{z}=\left\{\operatorname { s i n } \theta _ { 0 } \operatorname { c o s } \varphi _ { 0 } \left[A_{1} \cdot \sin \theta \cos \theta \cos \varphi+A_{2}\right.\right. \\
& \cdot \sin \theta \cos \theta \cos \varphi]+\sin \theta_{0} \sin \varphi_{0}\left[A_{1}\right. \\
& \left.\cdot \sin \theta \cos \theta \sin \varphi+A_{2} \cdot \sin \theta \cos \theta \sin \varphi\right]+\cos \theta_{0} \\
& \left.\cdot\left[A_{1} \cdot \cos ^{2} \theta-A_{2} \cdot \sin ^{2} \theta-\frac{\partial^{2}}{\partial t^{2}}\right]\right\} \frac{\mu}{4 \pi r} p\left(t-\frac{r}{c}\right) \text {, } \\
& A_{1}=\left(\frac{2 c^{2}}{r^{2}}+\frac{2 c}{r} \frac{\partial}{\partial t}+\frac{\partial^{2}}{\partial t^{2}}\right) A_{2}=\left(c \frac{\partial}{\partial t}+\frac{c^{2}}{r}\right) .
\end{aligned}
$$




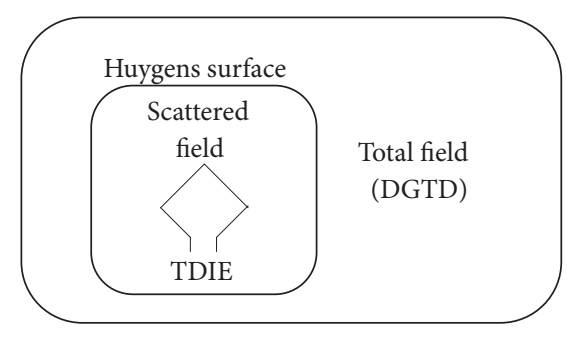

(a) Hybrid method setup

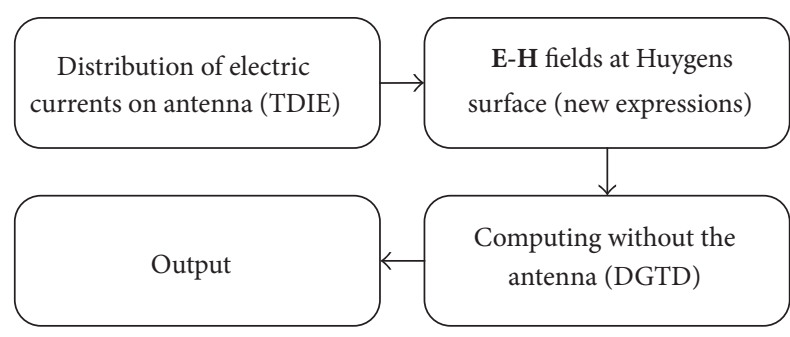

(b) Computing procedures

FIgURE 2: The hybrid TDIE/DGTD method.

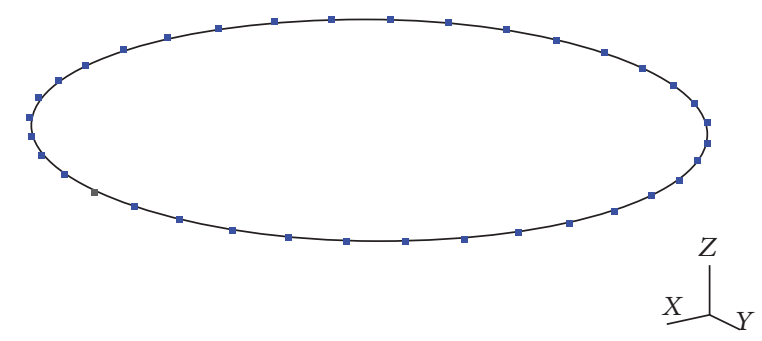

FIGURE 3: Circular antenna (divided into 35 straight lines).

\section{The Hybrid Method \\ Combining DGTD and TDIE for Wire Antenna-Dielectric Interaction}

TDIE is widely used for analyzing thin-wire antenna (radiation on scattering) problems, but it is difficult to deal with interaction of the antenna and complexity media. The TDIE/FDTD method and the FDTD/FETD/TDIE method have been proposed for complex electromagnetic problems [9, 10]. Discontinuous Galerkin Time-Domain (DGTD) method [11-15] inherits from Finite Element Time-Domain (FETD) the advantage of unstructured grids without solving large linear systems. DGTD is more flexible than Finite Difference Time-Domain (FDTD) method in geometry modeling, but like FDTD, it also becomes resource-consuming dealing with thin-wire structures.

Gao et al. proposed the TDIE/DGTD method [16]; in our study, the currents on the antenna can be easily obtained by TDIE. These currents are used to calculate $\mathbf{E}-\mathbf{H}$ fields on Huygens surface which yields the same radiating fields. The E-H fields are deduced from our new expressions, as shown in Figure 2.

4.1. A Circular Antenna. The antenna is parallel to the XOY plane, the radius of the antenna is 0.5 meter, the excitation wavelength is 0.5 meter, and the time harmonic current is excited at every line element (Figure 3). The normalized results of TDIE/DGTD with our new expressions are shown in Figures 4(a) and 4(b); a good agreement is observed between our algorithm (circle) and analytical solution (solid line) [17].

4.2. A Right Angle Antenna with Radome. We analyzed a right angle antenna located in the center of a dielectric ellipsoidshell radome (semiprincipal axes $a=1$ meter, $b=1$ meter, and $c=0.5$ meters); the thickness of the radome is 0.25 meters, the relative electric permittivity is 3.0 , and the relative permeability is 1.0 . The antenna is parallel to the plane $Y O Z$ (Figure 5); each arm of the antenna is 0.2 meters long. Antenna is excited by time harmonic current whose wavelength is 0.5 meters. The results of TDIE/DGTD and DGTD are shown for comparison in Figure 6; a good agreement is observed between two results.

4.3. A Circular Antenna with Radome. In this example, the antenna in the example of Section 4.2 is replaced with the circular antenna in the example of Section 4.1; excitation parameters of antenna are the same as the example of Section 4.2 (Figure 7).

Figure $8(\mathrm{a})$ is the normalized radiation pattern of circular antenna with radome. It is obvious that the main lobe of the pattern with radome is more intensive than without radome, as Figure 8(a) shows.

Next, the antenna is excited by a voltage pulse source, as shown in Figure 9, the distribution of currents on antenna is derived from TDIE. The voltage source is defined as

$$
V=\exp \left(\frac{t-t_{0}}{\tau}\right)^{2} \quad\left(\tau=2 \mathrm{~ns}, t_{0}=8 \mathrm{~ns}\right)
$$

Follow the steps in Figure 2(b); the snapshots in Figure 11 of the absolute value of electric field $|\mathbf{E}|$ are obtained from the hybrid TDIE/DGTD method. As shown in Figure 11(a), the electric field density is higher in the half-space $x>0$, because the voltage source is located at the cross point of antenna and $+x$ axis. The reflection radiation caused by radome can be seen in Figures 11(b), 11(c), and 11(d). The current waveform of source point is shown in Figure 10; the current tends to a value that is not zero. 


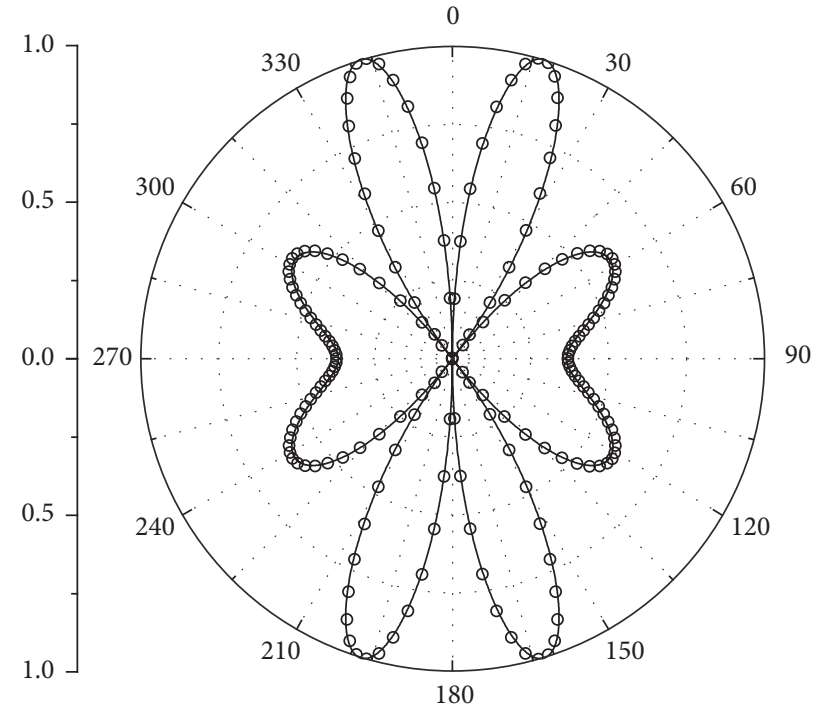

- TDIE/DGTD

Analytical solution

(a) The radiation pattern of $X O Z$ plane

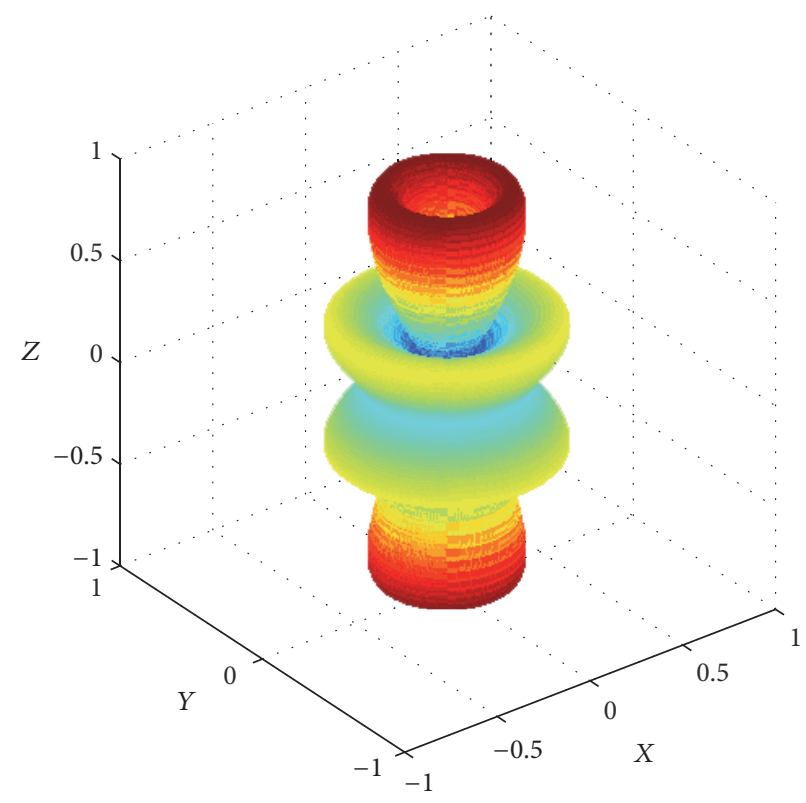

(b) Three-dimensional radiation pattern

FIGURE 4: The radiation pattern of a circular antenna.

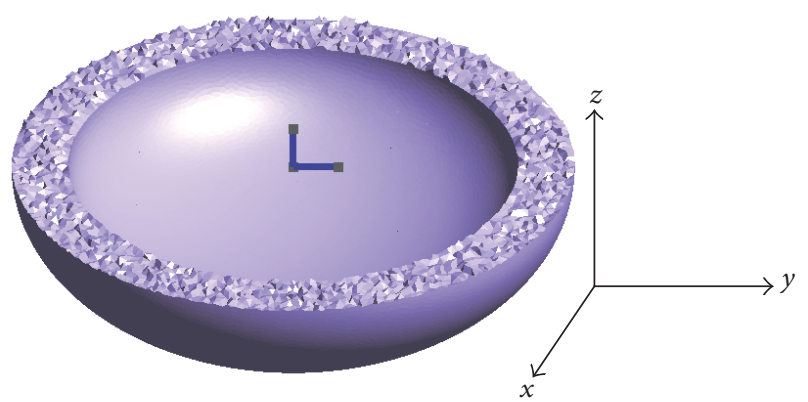

FIgURE 5: Sectional view grid.

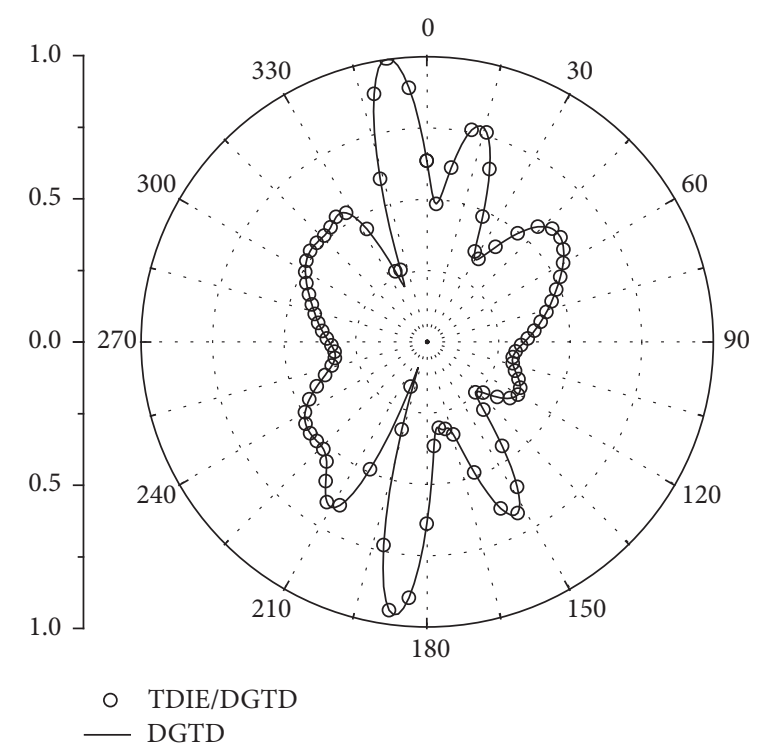

FIGURE 6: The radiation pattern of $Y O Z$ plane. 


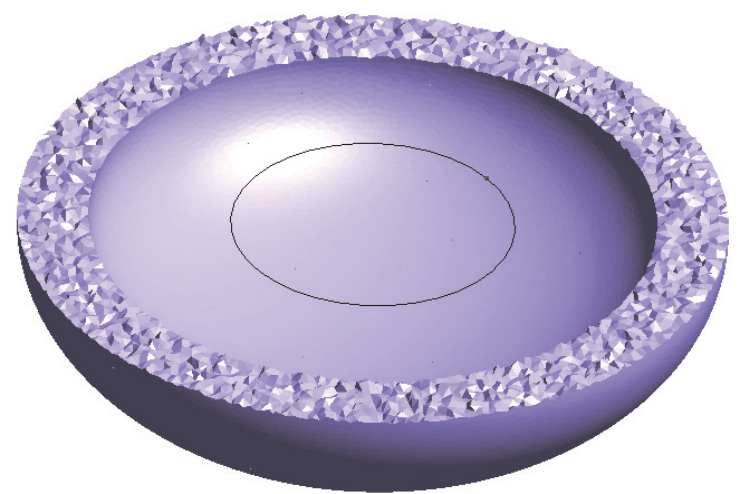

FIgURE 7: Sectional view grid.

0

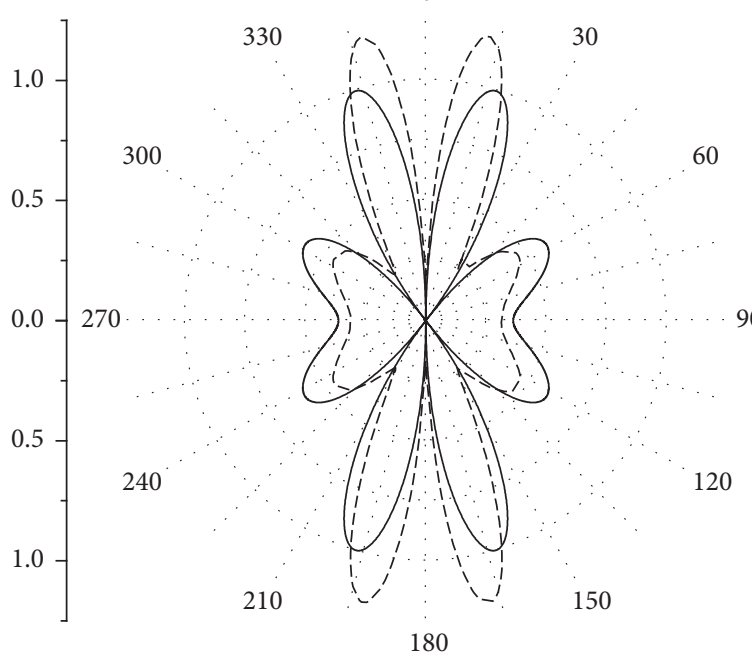

Antenna with radome

Antenna without radome

(a) The radiation pattern of $X O Z$ plane

60

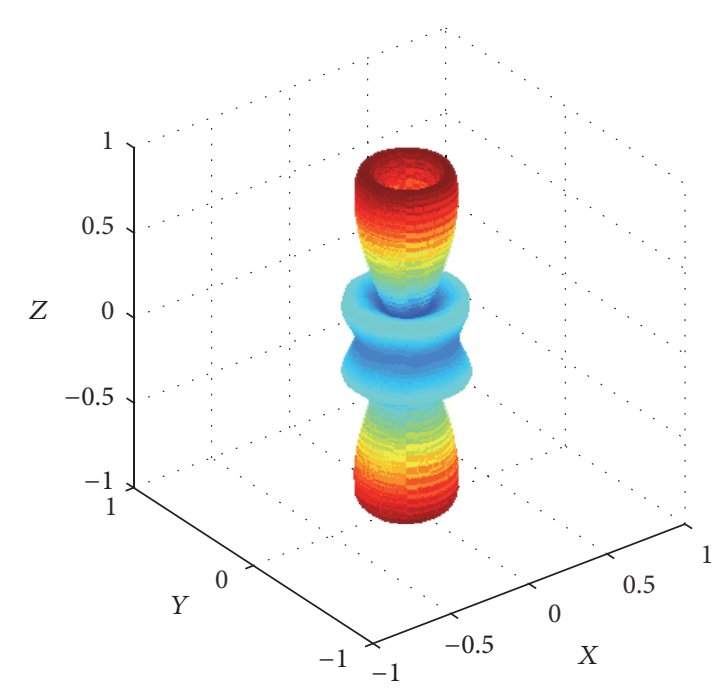

(b) Three-dimensional radiation pattern

FIGURE 8: The radiation pattern of circular antenna with radome.

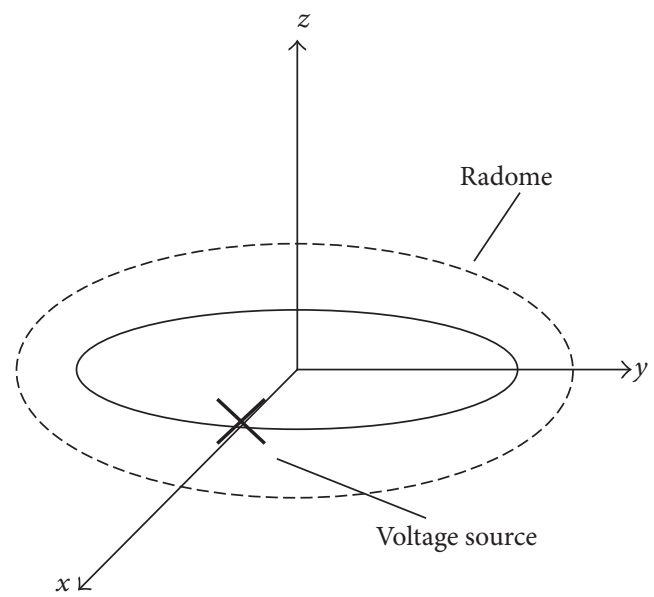

FIGURE 9: Voltage source. 


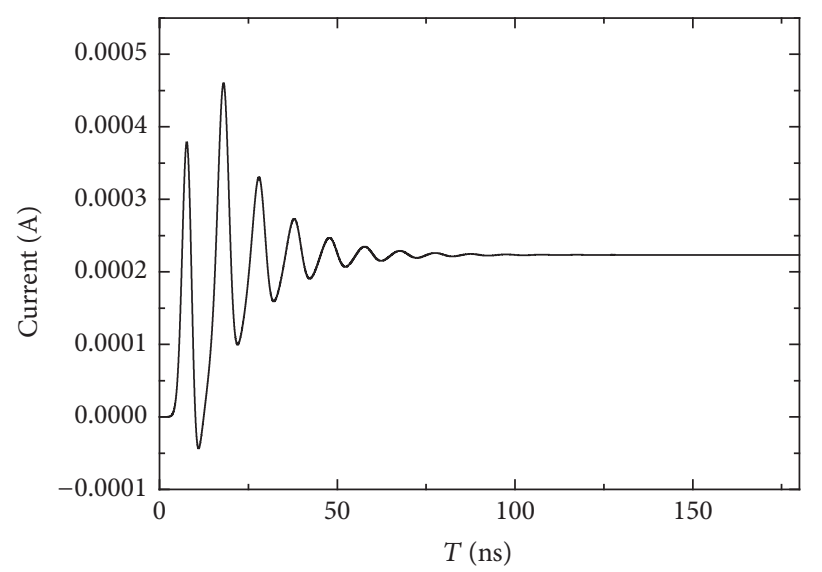

Current of source point

FIGURE 10: Current of source point.

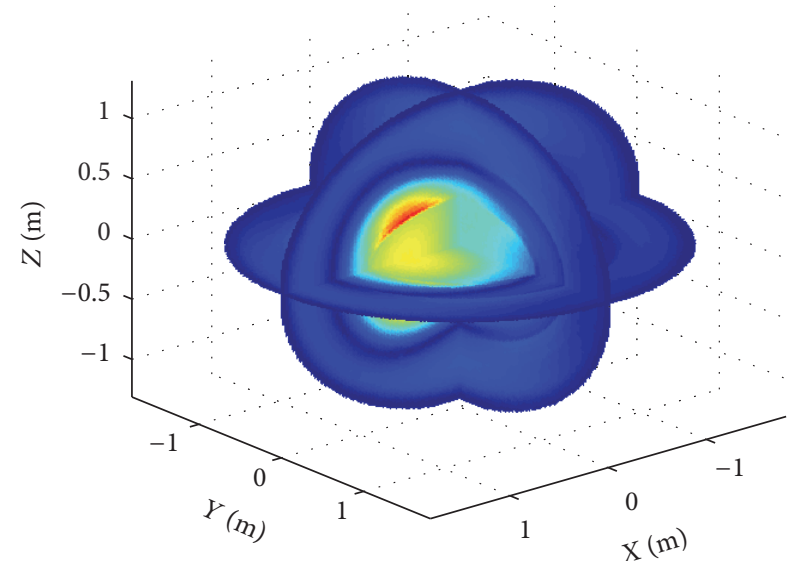

(a) 1000th time-step

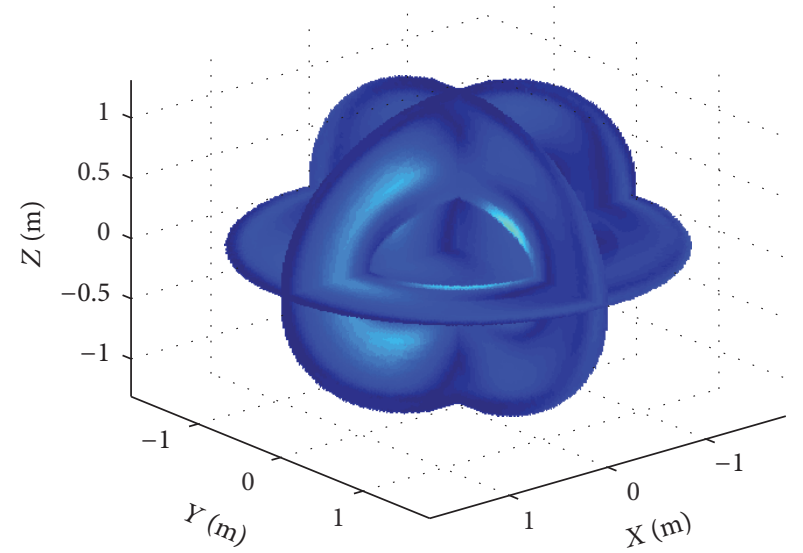

(c) 2200th time-step

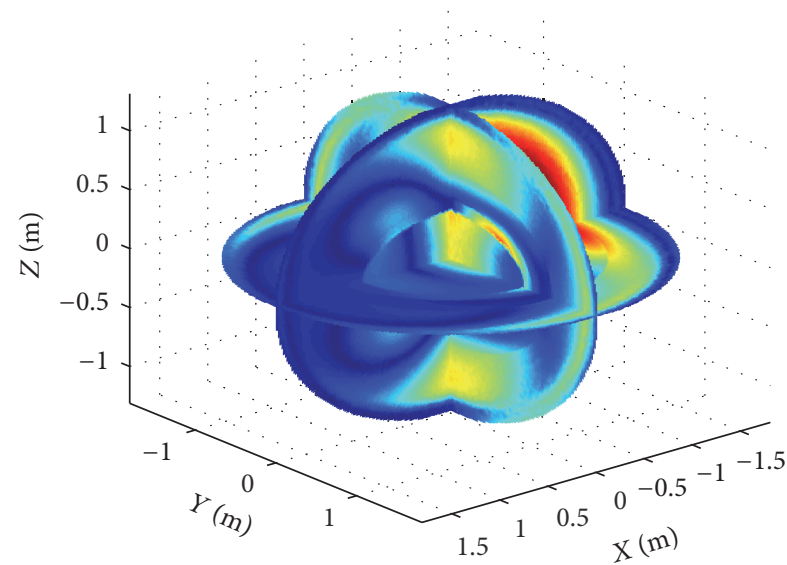

(b) 1600th time-step

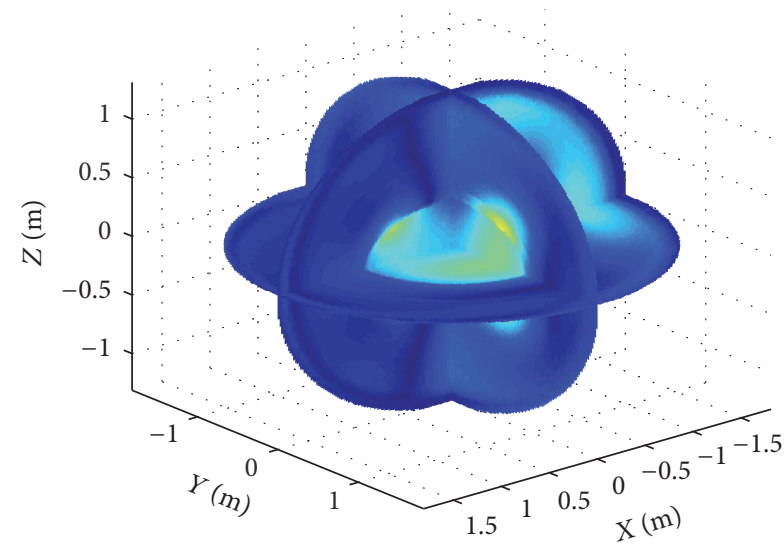

(d) 2800th time-step

FIgURE 11: Snapshots of $|\mathbf{E}|$. 


\section{Conclusion}

The analytical expressions of time-domain near fields of an arbitrarily oriented electric dipole are derived in this paper; then the expressions are used in hybrid TDIE/DGTD method for analysis of circular antenna with radome. Our study provides a new way to study the time-domain radiation problems of complex medium and structure.

\section{Conflicts of Interest}

The authors declare that there are no conflicts of interest regarding the publication of this paper.

\section{Acknowledgments}

This work was supported by the National Natural Scientific Foundation of China $(61571348 ; 61231003 ; 61401344)$.

\section{References}

[1] J. D. Jackson, Classical Electrodynamics, Wiley, New York, NY, USA, 1999.

[2] D. Y. Wu, Theoretical Physics, Science Press, Beijing, 2010.

[3] S. J. Zhang, Engineering Electromagnetic Theory, Science Press, Beijing, 2009.

[4] D. B. Ge and B. Wei, Electromagnetic Wave Theory, Science Press, Beijing, 2011.

[5] L.-X. Yang, D.-B. Ge, and B. Wei, "FDTD/TDPO hybrid approach for analysis of the EM scattering of combinative objects," Progress in Electromagnetics Research, vol. 76, pp. 275-284, 2007.

[6] L. Cao and B. Wei, "Scattering of targets over layered half space using a semi-analytic method in conjunction with FDTD algorithm," Optics Express, vol. 22, no. 17, pp. 20691-20704, 2014.

[7] F. Wang, B. Wei, and L. Q. Li, "Newmark method for finitedifference time-domain modeling of wave propagation in frequency-dispersive medium," Acta Physica Sinica, vol. 63, no. 10, pp. 389-394, 2014.

[8] B. Zhou, X. J. Hao, and X. Zheng, "Analysis of MoM/FDTD hybrid technique on shortwave antenna in complex environment," Information and Electronic Engineering, vol. 8, no. 6, pp. 669-672, 2010.

[9] A. Rubio Bretones, R. Mittra, and R. Gómez Martín, “A Hybrid Technique Combining the Method of Moments in the Time Domain and FDTD," IEEE Microwave and Guided Wave Letters, vol. 8, no. 8, pp. 281-283, 1998.

[10] A. Monorchio, A. Rubio Bretones, R. Mittra, G. Manara, and R. G. Martín, "A hybrid time-domain technique that combines the finite element, finite difference and method of moment techniques to solve complex electromagnetic problems," Institute of Electrical and Electronics Engineers. Transactions on Antennas and Propagation, vol. 52, no. 10, pp. 2666-2674, 2004.

[11] I. Perugia and D. Schötzau, "The $h_{p}$-local discontinuous Galerkin method for low-frequency time-harmonic Maxwell equations," Mathematics of Computation, vol. 72, no. 243, pp. 1179-1214, 2003.

[12] P. Houston, I. Perugia, and D. Schötzau, "hp-DGFEM for Maxwell's equations," in Numerical Mathematics and Advanced Applications, pp. 785-794, Springer, Milan, Italy, 2003.

[13] B. Cockburn, F. Li, and C.-W. Shu, "Locally divergence-free discontinuous Galerkin methods for the Maxwell equations,"
Journal of Computational Physics, vol. 194, no. 2, pp. 588-610, 2004.

[14] A. Catella, V. Dolean, and S. Lanteri, "An implicit discontinuous Galerkin time-domain method for two-dimensional electromagnetic wave propagation," COMPEL. The International Journal for Computation and Mathematics in Electrical and Electronic Engineering, vol. 29, no. 3, pp. 602-625, 2010.

[15] S. G. Garcia, M. F. Pantoja, C. M. De Jong Van Coevorden, A. R. Bretones, and R. G. Martín, "A new hybrid DGTD/FDTD method in 2-D," IEEE Microwave and Wireless Components Letters, vol. 18, no. 12, pp. 764-766, 2008.

[16] S. P. Gao, Y. L. Lu, and Q. S. Cao, "Hybrid method combining DGTD and TDIE for wire antenna-dielectric interaction," Applied Computational Electromagnetics Society Journal, vol. 30, no. 6, pp. 677-681, 2015.

[17] J. D. Kraus and R. J. Marhefka, Antenna for All Applications, McGraw Hill, Upper Saddle River, NJ, USA, 2002. 


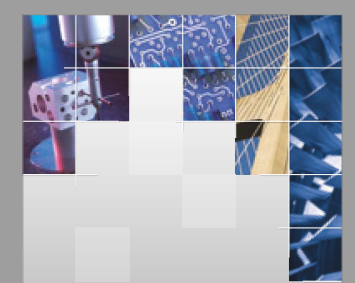

\section{Enfincering}
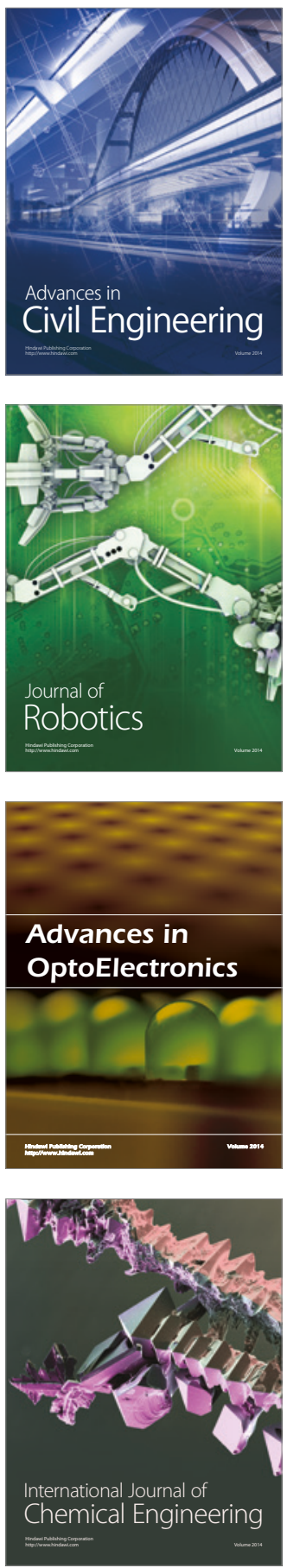

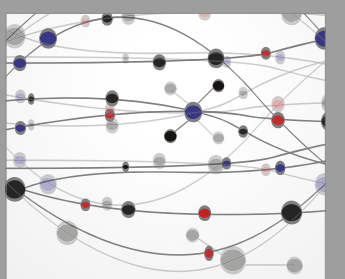

The Scientific World Journal

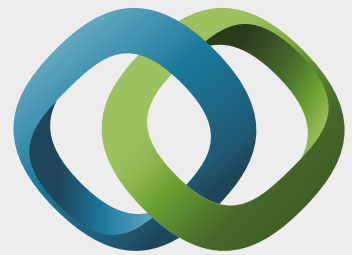

\section{Hindawi}

Submit your manuscripts at

https://www.hindawi.com
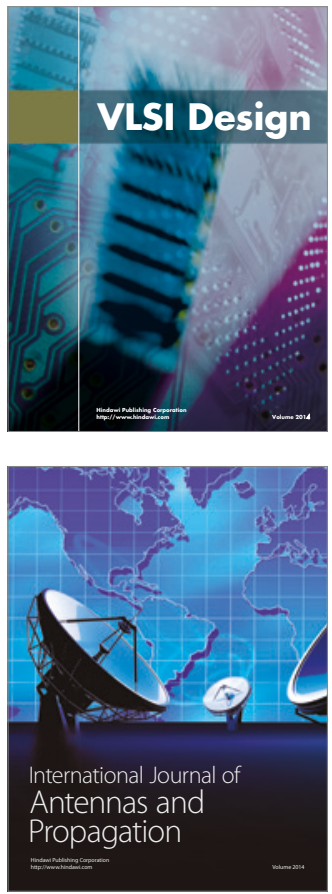

\section{Rotating}

Machinery
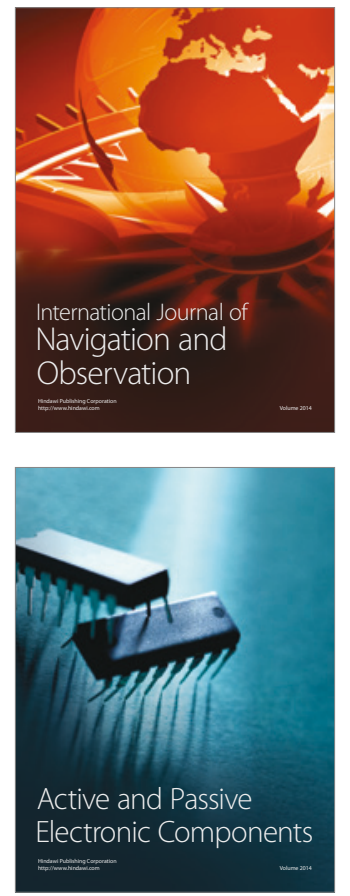
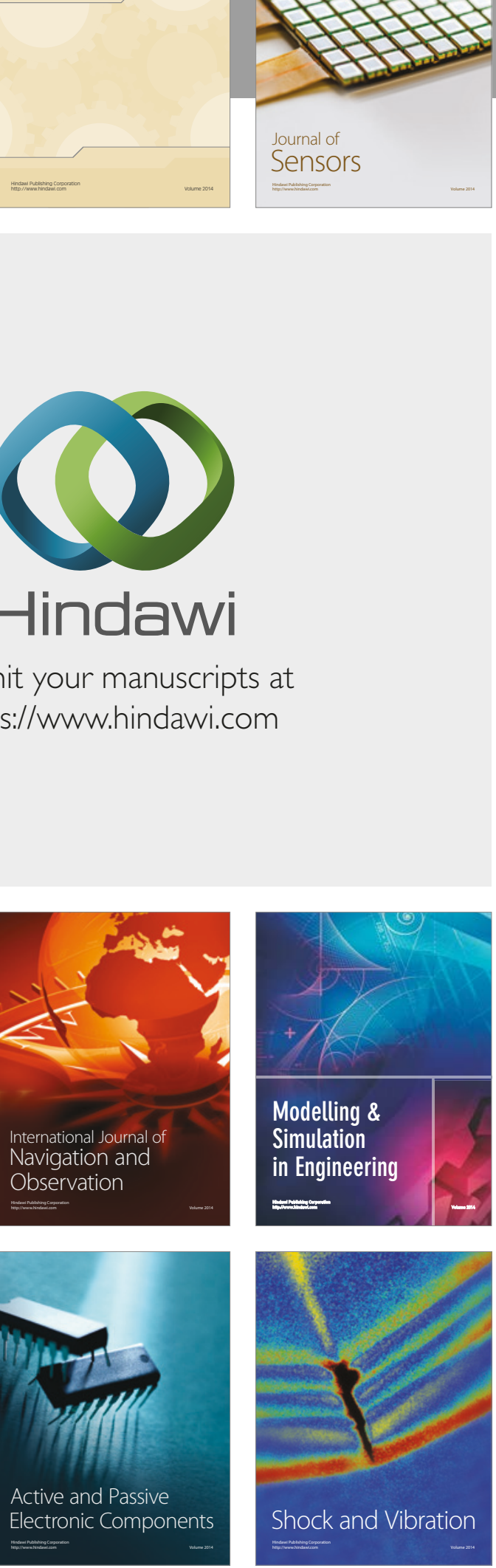
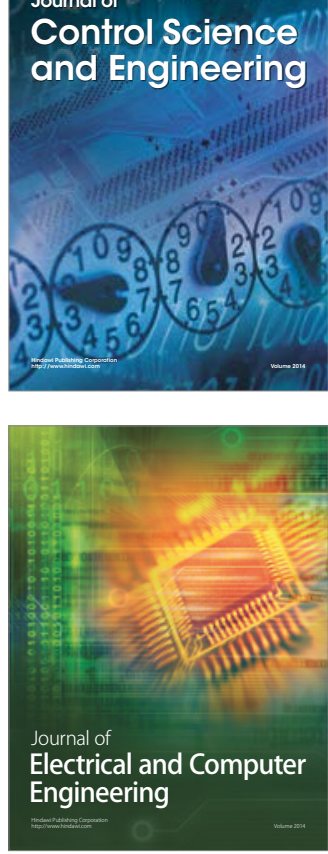

Distributed

Journal of

Control Science

and Engineering
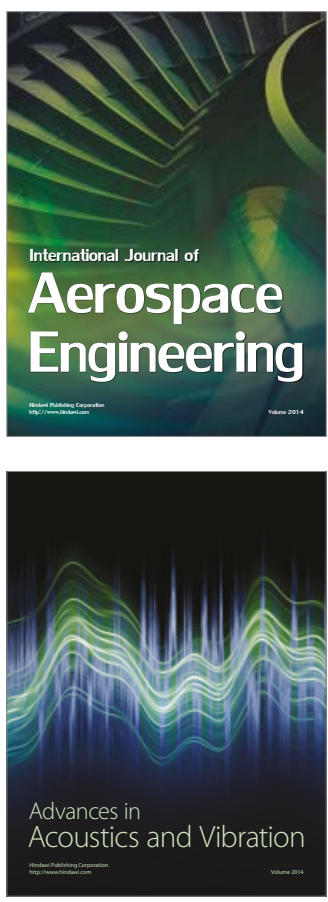

Sensor Networks 